
cultures

Les cahiers de l'Acedle

9-2 | 2012

Notions en questions - Les littératies

\title{
Les littératies : une Notion en Questions en didactique des langues ( $\mathrm{NeQ}$ )
}

Muriel Molinié et Danièle Moore

\section{OpenEdition}

Journals

Édition électronique

URL : http://journals.openedition.org/rdlc/2757

DOI : $10.4000 /$ rdlc. 2757

ISSN : 1958-5772

Éditeur

ACEDLE

Référence électronique

Muriel Molinié et Danièle Moore, «Les littératies : une Notion en Questions en didactique des langues (NeQ) », Recherches en didactique des langues et des cultures [En ligne], 9-2 | 2012, mis en ligne le 10 septembre 2018, consulté le 20 avril 2019. URL : http://journals.openedition.org/rdlc/2757 ; DOI : $10.4000 /$ rdlc. 2757

Ce document a été généré automatiquement le 20 avril 2019

\section{(ब) $\Theta \Theta$}

Recherches en didactique des langues et des cultures is licensed under a Creative Commons AttributionNonCommercial-NoDerivatives 4.0 International License 


\title{
Les littératies : une Notion en Questions en didactique des langues (NeQ)
}

\author{
Muriel Molinié et Danièle Moore
}

\section{Les littératies : une notion en questions en didactique}

1 S'inscrivant dans la tradition des Journées $\mathrm{NeQ}$ (Notions en Questions), la Journée organisée le 28 janvier 2011 à l'université de Cergy-Pontoise en collaboration avec le Diltec de l'université Paris 3 - Sorbonne Nouvelle et l'ACEDLE, interrogeait une notion largement circulante, mais délicate à saisir : celle de littératies. La journée d'étude rassemblait alors plusieurs contributeurs européens et nord-américains issus de divers champs disciplinaires et travaillant sur une variété de contextes. La journée mettait ainsi en dialogues sur une formule intervention - réaction: Donna Patrick et Gabriele Budach, et Aïssatou Mbodj-Pouye ; Béatrice Fraenkel et Jacques David ; Jeannine Gerbault et MarieClaude Penloup; Diane Dagenais et Martine Marquilló Larruy. Jean-Louis Chiss était le grand témoin de cette journée et en assurait clôture et synthèse.

2 Faisant suite à cette Journée, la publication de ce nouveau Numéro des Cahiers de l'Acedle rassemble des contributions sensiblement redistribuées. L'esprit en reste toutefois similaire : favoriser des rencontres intellectuelles autour de l'interrogation d'une notion complexe, comprise et souvent appréhendée de manière différente de part et d'autre du monde académique. D'où notre choix d'un pluriel pour ce concept polyvoque.

3 À cela, plusieurs raisons.

4 Rappelons tout d'abord que, dans le contexte français :

le potentiel didactique de la notion de littératie, instaurant une forme de continuité de la connaissance des systèmes d'écriture à la construction des discours est loin d'être négligeable. Chercher les formes de relation entre les dimensions culturelles et cognitives dans les actes de lire et d'écrire, distinguer la scripturalité (les écrits) et la textualité (les textes), reprendre sur d'autres bases les dichotomies culture de 
l'écrit / culture de l'oral, articuler les travaux entre les pratiques scolaires et extrascolaires (familiales et sociales) constituent des chantiers qui concernent fondamentalement la didactique des langues (...). Ces recherches sont, en effet, d'autant plus utiles à la didactique qu'elles unissent des perspectives techniques et culturelles où les variations éducatives jouent un rôle déterminant (Chiss, in : Dictionnaire de didactique du français langue étrangère et seconde, 2003, nous soulignons ; voir aussi Jaffré, 2004).

Dans un numéro récent de Recherches et applications, le Français dans le Monde, Bouchard et Kadi (2012) définissent la notion de littératie comme "globalement, au delà des savoirfaire rédactionnels et lecturels, une culture de l'écrit, qui permet à qui la possède de maîtriser le temps, l'espace, le monde d'une manière spécifique » (p. 11). On voit ici aussi se profiler l'idée de variabilité des expériences littératiées, en lien à des valeurs différentes et différentes façons de concevoir le monde et de le conceptualiser, notamment sous forme scripturo-graphique. Les pratiques littératiées répondent à une variété d'objectifs sociaux et sont évolutives dans le temps et l'espace, elles sont liées à des valeurs, des représentations, des relations institutionnelles et sociales (Dabène, 1988 ; Collins et Blot, 2003 ; Barré-de Miniac, Brissaud et Rispail, 2004 ; Goody, 2007 ; Lahire, 2008 ; voir aussi Dagenais \& Moore, 2008) : "Y sont incluses la conscience qu'ont les individus de la littératie, la façon dont ils en parlent - leurs constructions, leurs discours - et les significations qu'ils lui donnent » (Barton et Hamilton, 2010 : 46). La littératie, écrit encore Menigoz (2001: 133), c'est «l'ensemble des inscriptions, des listes, des répertoires, des textes en tout genre, de tout écrit qui permet une conservation ou une transmission de savoirs et d'informations. Elle induit la compréhension ambiante de ce que l'écrit peut permettre comme savoir, et l'accès à ces savoirs, et ce que cela implique comme logique mentale et comportements de la pensée » (voir aussi Moore, 2006). En France, le questionnement autour de la littératie reste très profondément attaché à une réflexion autour du lien entre écriture et école et, partant, interroge la complexité des relations entre langue, culture et cognition et la notion de compétence (Dabène, 1990). Dans une perspective communicationnelle et culturellement marquée des usages, il convient de s'interroger alors, notamment à l'école, «sur les formes de la constitution et de l'appropriation des savoirs, les techniques requises pour ce faire et la maitrise des espace graphiques, l'ensemble de ce que Goody nomme souvent une 'technologie de l'intellect" "(Chiss, 2011: 6; Goody 1968a et b/2006; voir aussi Chiss, 2004 et 2012; Reuter, 2006). Ces espaces graphiques incluant tout autant, rappelle Chiss, le tableau noir, l'écran d'ordinateur ou le cahier, que la structuration à et par l'écrit des contenus disciplinaires, notamment sous la forme des manuels, etc.

Les études sur les littératies invitent donc, depuis plusieurs années, les didacticiens à un élargissement du champ de leur réflexion sur les pratiques sociales du lire-écrire en en sollicitant des dimensions à la fois cognitive, linguistique, psycholinguistique et sociale, sociolinguistique, anthropologique, économique et historique. En témoignent le nombre de numéros spéciaux consacrés à des thématiques proches, telles que, pour n'en citer que quelques-unes ces dernières années, Lidil (La littéracie. Vers de nouvelles pistes de recherche didactique, 2003, numéro 27, coordonné par Christine Barré-de Miniac) ; Pratiques (La littératie. Autour de Jack Goody, 2006, numéro 131/132, coordonné par Mohamed Kara et Jean-Marie Privat) ; Glottopol (Regards sur l'internet, dans ses dimensions langagières. Penser les continuités et discontinuités, En hommage à Jacques Anis, 2007, numéro 10, coordonné par Isabelle Pierozack); The Canadian Modern Language Review/La revue canadienne des langues vivantes (Multilingual Literacies/Littératies plurilingues, 2008, volume 65(1), coordonné par 
Diane Dagenais et Heather Lotherington), Langage et Société (Les New Literacy Studies, 2010, numéro 133, coordonné par Béatrice Fraenkel B. et Aïssatou Mbodj); Recherches et applications, Le Français dans le Monde (Didactiques de l'écrit et nouvelles pratiques d'écriture, 2012, numéro 51, coordonné par Robert Bouchard et Latifa Kadi).

7 La thématique n'est ainsi pas nouvelle dans le champ de la didactique du français. On rappellera notamment pour notre propos ici le numéro 4 de la revue Cahiers du français contemporain, coordonné en 1997 par Martine Marquilló Larruy, dans lequel Jacques Anis interroge la lecture des "visuo-textes » et où se posent alors des questions par rapport aux nouvelles formes d'écriture, de situations et d'outils : le traitement de texte (Sylvie Plane), le fax (Pierre Delcambre), le télétravail (Françoise Rouard) mais aussi encore les albums de jeunesse (Francis Grossmann), les manuels scolaires (Gérard Vigner), la poésie urbaine (Jacqueline Billiez) ou encore le récit de migrations, à l'oral/à l'écrit en groupes de pairs (Muriel Molinié). Martine Marquilló Larruy notait alors dans son introduction :

Si on a pu craindre un jour que les images et les facilités de communications orales réduiraient l'écrit à la portion congrue, on sait aujourd'hui qu'il n'en est rien : la civilisation Gutenberg loin d'être mortelle est plus galaxique et vigoureuse que jamais. La passion qui anime les débats autour de l'illetrisme, l'émergence et la vitalité du concept de littératie traduisent non seulement la place de choix qu'occupent les écrits dans notre société mais aussi l'élévation des exigences sociales en matière d'écriture (1997 : 9).

8 C'est le développement du concept de communication qui a permis, nous dit encore Martine Marquilló Larruy, de redynamiser les approches de l'écrit en permettant une meilleure compréhension de l'inscription sociale des textes et, donc, des situations d'écriture (p. 11) et, partant, à élargir l'étude de ces situations pour englober aussi une diversité de pratiques de tous les jours: de la liste de courses à faire, aux fascicules des services publics (Lapeyre, 1993) jusqu'aux aux épitaphes de cimetière (Jean-Marc Colletta, 1990) ou, plus récemment, les messages textuels (ou tchats) que l'on échange en temps réel sur les sites internet (Pierozack, 2003). Ces pratiques de toutes formes constituent pour Michel Dabène, des "écrits (extra)ordinaires" (Dabène, 1990) qu'il convient de décrire et d'étudier, dans des perspectives d'amélioration de l'action didactique. C'est dans un même esprit que Reuter et Penloup (2001) s'intéressent aux pratiques extrascolaires de lecture et d'écriture des élèves, à la fois dans l'espoir de se défaire de «l'ethnocentrisme lettré » (Reuter, 2001: 12 citant Grignon et Passeron, 1989) tout en restant vigilant sur «[...] le risque pour les éléves à masquer, peu consciemment, peu volontairement, sans doute, les enjeux sociaux, identitaires, culturels de ces pratiques » (Reuter, 2001 : 17, citant Bautier et Bucheton, $1997:$ 19).

9 Nous nous proposons ici de poursuivre en ce sens, en considérant à notre tour, les pratiques sociales du lire-écrire comme un continuum entre différents contextes et conditions de développement (familial, scolaire, sociétal) et sous diverses formes (scripturales et graphiques), depuis les premiers apprentissages jusqu'aux pratiques les plus élaborées, dans une ou plusieurs langues ou variétés de langues et selon diverses modalités, incluant le visuel et le digital.

10 Mais notre projet ne s'arrête pas là. Car il s'enrichit du fait que cette notion élastique, pour l'essentiel emprunté aux travaux anglo-saxons, subit des passages transcontinentaux et d'une langue à l'autre, véhiculant d'une rive à l'autre des emplois pluriels : nouvelles littératies, multilittératies, littératies plurilingues, plurilittératies, etc. 
11 Or si la prolifération des termes invite d'abord à un travail de clarification, elle incite également à tenter de cerner les rapports entre certaines de ces dénominations : littératies visuelles, numériques, digitales, multimodales, littératies critiques, etc., dans le contexte anglophone et dans le contexte francophone.

\section{Présentation des contributions}

Dans le présent numéro, qui questionne par mises en regards, parfois décalés, la notion de littératies, nous avons cherché à mettre en évidence les zones de continuité et parfois de dissonances des débats. On verra ainsi (dans les trois premières contributions), que le foisonnement terminologique témoigne plus fondamentalement d'une discussion qui traverse le monde éducatif nord-américain sous la dénomination de pédagogies - et perspectives - critiques en éducation, dont les New Literacy Studies (ou Nouvelles études de la littératie) se font largement l'écho de part et d'autres de l'Atlantique.

C'est pourquoi nous avons choisi d'ouvrir le présent numéro avec la contribution de Diane Dagenais qui présente le développement de la littératie multimodale dans le paysage nord-américain, et plus spécifiquement canadien. Dans son article intitulé Littératies multimodales et perspectives critiques, elle examine les notions de littératies multiples, littératies plurilingues, répertoire plurilittératié, multimodalité et littératies multimodales qui circulent dans les écrits en anglais et dans quelques publications récentes en français au Canada. La discussion menée par l'auteure montre très clairement comment, dans une perspective critique, les recherches portant sur l'apprentissage des pratiques de littératie identifient des pistes pédagogiques équitables, c'est-à-dire offrant aux enfants d'origines linguistiques et culturelles diverses une plus grande variété de ressources sémiotiques pour s'exprimer, se représenter et développer leurs pratiques de communication dans une société de plus en plus hétérogène et technologisée. D. Dagenais étudie quelques données provenant d'une recherche menée sur un projet de production et d'échange de vidéos mené auprès d'enfants bilingues et plurilingues vivant dans différents pays afin d'illustrer quelles conditions matérielles et sociales facilitent - ou entravent - l'apprentissage. À travers une innovation pédagogique fondée sur les littératies multimodales, il s'agit donc d'une part, d'améliorer les conditions matérielles et sociales facilitant les apprentissages en contexte plurilingue et, d'autre part, de former de futurs enseignants à ce type d'approche.

Intitulé Littératie et multimodalité ici \& là-bas... En réponse à Diane Dagenais, le texte de Martine Marquilló Larruy poursuit cette entreprise de clarification de la notion de littératie multimodale. Elle passe tout d'abord en revue les diverses raisons (socioculturelles et historiques), qui ont rendu délicate l'appropriation des notions de littératie et de littératie multimodale dans le contexte hexagonal français. M. Marquilló Larruy analyse ensuite le débat qui confronte les tenants d'une littératie conçue comme une technologie de l'intellect et ceux qui donnent la prééminence aux conditions sociales de son ancrage. L'intérêt de cet article est donc premièrement de montrer en quoi et pourquoi les termes littératie et multimodalité renvoient, de part et d'autre de l'Atlantique, à des découpages disciplinaires différents, que ce soit dans le domaine de l'anthropologie de l'écriture, celui de la didactique des langues ou encore celui des "nouvelles" technologies. Mais l'auteure va plus loin en montrant également de façon précise comment le débat entre tenants d'une perspective «autonome » de la littératie et ceux qui la considèrent comme dépendante de son inscription sociale, est particulièrement 
stimulant pour modifier des représentations monolithiques des situations de littératie. M. Marquilló Larruy souligne, pour terminer, combien

la recherche a besoin de se nourrir de ces regards culturels croisés qui déstabilisent et interrogent nos certitudes « et affirme que »c'est précisément la connaissance affinée de la diversité des contextes avec la prise en compte des particularités qui y sont associées qui devrait permettre aux enseignants, aux décideurs des politiques linguistiques et éducatives une plus grande clairvoyance, une plus grande souplesse pour admettre la nécessité de réfléchir à des propositions élaborées ad hoc, pour chaque contexte considéré (M. Marquilló Larruy, infra).

Dans leur l'article intitulé Chaque objet raconte une histoire, Les pratiques de littératie chez des Inuit en milieu urbain, Donna Patrick et Gabriele Budach envisagent à leur tour la notion de littératie dans une perspective critique : celle d'éclairer les rapports entre processus de migration et pratiques locales de littératie. S'inscrivant dans le courant des New Literacy Studies, leur analyse porte en effet sur les pratiques de littératie de mobilité mises en oeuvre dans un centre communautaire, par des Inuit urbains à Ottawa au Canada. Pour cela, elles interrogent le rôle que jouent certains objets (pris dans des interactions), pour retracer des trajectoires de migration et relier différents mondes de vie. Les auteures montrent comment, au sein d'évènements de littératie, des objets peuvent jouer un rôle central comme ressource sémiotique assumant trois types de fonction. Premièrement, celle de rappeler des moments significatifs et de reconstruire un parcours individuel mis en lien avec les parcours d'autres membres de la communauté Inuit présents au cours de l'interaction (ce qui forme l'idée d'un parcours structuré à l'intérieur d'une communauté). Deuxièmement, la fonction de représenter des liens avec d'autres communautés mettant en lumière la construction de réseaux sociaux et familiaux qui ont joué un rôle clé dans la formation d'identité dans le passé. Troisièmement, la fonction de contextualiser des expériences ultérieures dans un contexte actuel: celui de la communauté urbaine unifiant des Inuit de différentes régions de l'Arctique. Dès lors, la recherche menée par Donna Patrick et Gabriele Budach sur les plurilinguismes et pour le développement communautaire interroge les pratiques d'une communauté autochtone (minoritaire) qui partage des concepts et des repères culturels différents de ceux véhiculés par la culture majoritaire. Les auteures concluent sur l'intérêt qu'il y a à reconnaitre l'émergence et la co-existence au sein d'une même société de plusieurs définitions de la littératie dans des contextes communautaires spécifiques. Connaitre ces définitions contextualisées doit en effet permettre de créer des ponts entre les normes de la société majoritaire et les communautés de migration afin de créer le "socle d'une compréhension mutuelle » dont la littératie ne serait qu'une concrétisation parmi d' autres.

16 Les deux contributions suivantes interrogent premièrement, la place de la littératie numérique dans les compétences à construire pour les citoyens du $21^{\text {ème }}$ siècle, et deuxièmement, les changements que produisent les littératies numériques en didactique du français écrit. Après avoir effectué une synthèse de cette notion en s'appuyant sur les travaux les plus récents, l'article de Jeannine Gerbault intitulé Littératie numérique, Les nouvelles dimensions de l'écrit au $21^{\text {ième }}$ siècle, aborde quelques questions relatives aux rapports entre littératie numérique et littératie traditionnelle - sur le plan de l'acquisition et sur celui des pratiques - ainsi qu'à son articulation avec la littératie extrascolaire. J. Gerbault analyse cette notion sous trois aspects: compétences de communication, compréhension de l'impact des technologies et des médias dans le 
monde qui nous entoure, et compréhension de la façon dont les technologies peuvent être utilisées dans les apprentissages formels ou informels.

Dans sa Réponse à Jeannine Gerbault, Marie-Claude Penloup interroge les littératies numériques sous l'angle de leurs enjeux pour la didactique de l'écriture-lecture. La révolution numérique a en effet introduit des écrits d'un nouveau genre ainsi que de nouveaux gestes liés à l'écrit qu'il s'agit pour l'apprenant de maitriser. Elle fournit, dans le même temps, de nouveaux outils pour l'enseignement-apprentissage de l'écriture-lecture. C'est dans ce double cadre que sont envisagées les questions posées à la didactique du français écrit par les littératies numériques. L'accent est mis sur le changement de posture qu'impliquent, chez l'enseignant, ces nouvelles formes de littératie et sur le gain que peut alors constituer leur irruption dans le cadre scolaire pour l'ensemble de la réflexion sur la didactique du français écrit, qu'il soit ou non électronique.

Le dernier mot est donné à Jean-Louis Chiss, qui, s'il nous invite à poursuivre dans le sens d'une sociologie (critique) des pratiques culturelles, nous enjoint simultanément à ne pas appauvrir la notion de littératie par un déplacement s'éloignant selon lui de plus en plus des problématiques didactiques posées par la/les culture(s) de l'écrit. Revenant sur l'apport central des travaux de Goody, il en rappelle deux points essentiels pour la réflexion didactique: «(...) le passage de la textualité à la scripturalité d'une part et, d'autre part, l'inscription de la littératie dans les cultures linguistiques et éducatives » ( infra). D'accord, nous dit encore J.-L. Chiss, pour une pluralisation des littératies dans un contexte d'interrogations sur les formes, la diversité culturelle et les espaces de leurs pratiques et leurs usages, de la famille, l'école, à la rue ou au monde du travail. Mais JeanLouis Chiss nous invite à rester critique sur le degré de pertinence d' "une pensée dualiste qui ferait des 'littératies locales' des lieux de contre-pouvoir » (infra) et d'une nouvelle forme d'idéologisation ${ }^{1}$.

La substitution du terme littérac(t)ie dans les travaux ne permet-elle alors que de «revêtir d'oripeaux modernes» (Reuter, 2003) des notions antérieures, sans que cette substitution ne change fondamentalement la façon de réfléchir les liens entre pratiques, représentations et contextes? Les contributions qui suivent permettront de faire un point sur les apports d'une notion complexe, désormais largement circulante, mais peu encore, au final, caractérisée d'une manière permettant l'intégration de ses multiples composantes. Elles visent, surtout, à ouvrir un espace de dialogue entre chercheurs de différents horizons, géographiques et disciplinaires, pour construire et déconstruire une notion diversement habitée et contribuer, selon la tradition des Notions en Questions (NeO), à la réflexion dans le domaine de la didactique des langues.

\section{BIBLIOGRAPHIE}

Barré-De Miniac, C. (éd.) (2003.) La littéracie. Vers de nouvelles pistes de recherche didactique. Lidil, $\mathrm{n}$ - 27 (numéro entier).

Barré-De Miniac, C. (2003). « Présentation », Lidil, n² 27, pp. 5-10. 
Barré-De Miniac, C., Brissaud, C., Rispail, M. (éds.) (2004). La littéracie (Conceptions théoriques et pratiques d'enseignement de la lecture-écriture). Paris : L'Harmattan, Collection Espaces Discursifs.

Barré-De Miniac, C. (éd.)(1996). Vers une didactique de l'écriture. Pour une approche pluridisciplinaire. Bruxelles : de Boeck.

Barton D. \& Hamilton M. (2010). « La littératie : une pratique sociale », Langage et société, n 133, pp. 45-62.

Bautier, E. \& Bucheton, D. (1997). « Les pratiques socio-langagières dans la classe de français ? Quels enjeux? Quelles démarches?», Repères, $n^{\circ}$ 15, pp. 11-26.

Bouchard, R. \& Kadi, L. (2012). Didactiques de l'écrit et nouvelles pratiques d'écriture. Recherches et applications, Le Français dans le Monde, $\mathrm{n}^{\circ} 51$ (numéro entier).

Chiss, J.-L. (2004). « La littératie : quelques enjeux d'une réception dans le contexte éducatif et culturel français ». In Barré-de Miniac \& al. (éds.). Op.cité, pp. 43-52.

Chiss, J.-L. (2003). Article « Littératie ». In Cuq, J.-P. (éd.). Dictionnaire de didactique du français langue étrangère et seconde. Paris : Clé international.

Chiss, J.-L. (2011). « Littératie et didactique de la culture écrite », Forumlecture.ch, n 1, 1-12 (Première parution dans la revue Pratiques (2008), $\mathrm{n}^{\circ}$ 137/138, pp. 165-168. Disponible en ligne. http://www.forumlecture.ch/myUploadData/files/2011_1_Chiss.pdf

Chiss, J.-L. (2012). L'écrit, la lecture et l'écriture. Théories et didactiques. Coll. Enfances et langages. Paris : L'Harmattan.

Collins, J. \& Blot, R. (2003). Literacy and literacies. Text, Power and Identity. Cambridge : Cambridge University Press.

Dabène, M. (1988). «L'évaluation des écrits ordinaires : systèmes évaluatifs et stratification sociale - Sociolinguistique : évaluation et analyses du discours ", Cahiers du français des années 80 , no 3, pp. 59-87.

Dabène, M. (1990). « Les pratiques d'écriture, représentations sociales et itinéraires de formation : points de vue sociolinguistique et didactique », Éducation permanente, $\mathrm{n}^{\circ} 102,19$.

Dabène, M. (éd.)(1990). Des écrits (extra)ordinaires - Éléments pour une analyse de l'activité scripturale. Lidil, $\mathrm{n}^{\circ} 3$ (numéro entier).

Dagenais, D. \& Moore, D. (2008). « Représentations des littératies plurilingues et de l'immersion en français chez des parents chinois », Canadian Review of Modern Languages, vol. 65, n 1, pp. 11-32. Delbreilh, F. (2012). « Les notions de speech event et de literacy event dans l'ethnographie de la communication et les Literacy Studies ", Langage et société, n 139, pp. 83-101.

Fraenkel, B. \& Mbodj, A. (2010). «Introduction. Les New Literacy Studies, jalons historiques et perspectives actuelles ", Langage et Société, n 133, pp. 7-24.

Goody, J. (1968a, 2006). « La technologie de l'intellect », Pratiques, n 131/132, pp. 7-30.

Goody, J. (1968b, 2006). « Les conséquences de la littératie », Pratiques, n 131/132, pp. 31-68.

Goody, J. (2007). Pouvoirs et savoirs de l'écrit. Paris : La Dispute.

Grignon, C. \& Passeron, J.-C. (1989). Le savant et le populaire. Misérabilisme et populisme en sociologie et en littérature. Paris : Seuil.

Jaffré, J.-P. (2004). « La litéracie : histoire d'un mot, effets d'un concept ». In C. Barré-De Miniac, C. Brissaud, M. Rispail (éds.). Op.cité, p. 21-42. 
Lahire, B. (2008). La raison scolaire. École et pratique d'écriture, entre savoir et pouvoir. Rennes : Presses universitaires de Rennes.

Lapeyre, F. (1993). « Quand les services publics s'adressent aux usagers », dans Kahn, G. (coord.), Des pratiques de l'écrit, Le Français dans le Monde/Recherches et applications, numéro spécial, févriermars 1993, pp. 182-190.

Menigoz, A. (2001). Apprentissage et enseignement de l'écrit dans les sociétés multilingues : L'exemple du Plateau Dogon au Mali. Paris : L'Harmattan.

Moore, D. (2006). Plurilinguismes et école. Paris : Didier.

Pierozack, I. (2003). Le français tchaté. Une étude en trois dimensions - sociolinguistique, syntaxique et graphique, d'usages IRC (Internet Relay Chat). Thèse de Doctorat, Université d'Aix-Marseilles, 3 vol. , 1433p.

Reuter, Y. (2001). «La prise en compte des pratiques extrascolaires de lecture et d'écriture : problèmes et enjeux', Repères, $\mathrm{n}^{\circ} 23, \mathrm{pp}$. 9-31.

Reuter, Y. (2006). »À propos des usages de Goody en didactique. Éléments d'analyse et de discussion", Pratiques, $n^{\circ}$ 131/132, pp. 131-154.

Reuter, Y. \& Penloup, M.-C. (coord.)(2001). Les pratiques extrascolaires de lecture et d'écriture des élèves, Repères, $\mathrm{n}^{\circ} 23$ (numéro entier).

Street, B. (1993). Cross-cultural Approaches to Literacy. Cambridge : Cambridge University Press.

\section{NOTES}

1. On rappelera que le courant des New Literacy Studies développé au Royaume-Uni sous l'impulsion de Brian Street et ses collègues s'oppose, entre autres, à la vision jugée trop déterministe des travaux de Jack Goody (Street, 1993 ; Fraenkel \& Mbodj, 2010 ; Delbreilh, 2012). Pour un questionnement autour de cette lecture des travaux de Goody, voir notamment Chiss (2004, 2011 et infra), Reuter (2006) mais aussi Goody (2007).

\section{RÉSUMÉS}

Fidèle à la tradition des journées $\mathrm{NeQ}$ (Notions en Questions), ce numéro spécial des Cahiers de l'Acedle interroge la notion de littératie(s), un concept élastique, pour l'essentiel emprunté aux travaux anglo-saxons mais dont les passages transcontinentaux et d'une langue à l'autre, demandent d'en éclairer les emplois pluriels. On s'interrogera plus précisément sur les représentations du développement littératié et les implications qui y sont associées, dans la sphère didactique en particulier.

True to the tradition of the "journées $\mathrm{NeQ}$ » (Notions in Question in Didactics), this special edition of the Cahiers de l'Acedle examines understanding of literacy/ies, an elastic and transdisciplinary notion, mostly borrowed from anglophone research, and explores how and why transcontinental passages from one language to another require explanations of its plural uses. 
We will be looking closely at the beliefs surrounding the situdated development of literacies and their implications, particularly in the realm of didactics.

\section{INDEX}

Keywords : literacy, culture of writing, language didactics

Mots-clés : littératie, culture de l'écrit, didactique des langues

\section{AUTEURS}

\section{MURIEL MOLINIÉ}

Université de Cergy-Pontoise, EA 1392, CRTF-LaSCoD, Diltec et GIS Pluralité des Langues et des Cultures (France.)

Muriel MOLINIÉ est maître de conférences à l'université de Cergy-Pontoise (UCP), membre du Centre de Recherche Textes et Francophonies/LaSCoD, du Diltec et du GIS Pluralités linguistiques et culturelles. Elle dirige le Centre international langue française et action culturelle (CILFAC) et la formation FLE/S de l'UCP. Elle enseigne la didactique des langues et du plurilinguisme, l'anthropologie de la diversité culturelle, les méthodes d'enseignement du français oral. Le développement de pratiques biographiques pluri-littératiées (récits de formation, biographies langagières, dessins et entretiens réflexifs), et la co-construction de Portfolios contextualisés sont devenus ses principaux outils de formation-action réflexive aux langues et aux cultures. La finalité des recherches-actions qu'elle coordonne est d'accompagner, de comprendre et de décrire les négociations identitaires vécues et verbalisées dans les langues de la migration, par les étudiants en mobilité, dans des contextes de formation internationale, via des approches multi-modales (vidéo, dessins, textes).

muriel.molinie[at]u-cergy.fr http://www.u-cergy.fr/fr/ molinie Université de Cergy-Pontoise, Bureau 004, Bd. du Port, 95000 Cergy-Pontoise.

\section{DANIÈLE MOORE}

Simon Fraser University, Vancouver (Canada) et Diltec, Université Paris 3 - Sorbonne Nouvelle (France)

Danièle Moore est professeur titulaire à la Faculté d'Éducation de l'Université Simon Fraser et directeur de recherche à l'université Paris 3 - Sorbonne Nouvelle (DILTEC, ED268). Ses recherches en sociolinguistique, en didactique des langues et en acquisition couvrent l'étude des plurilinguismes sociaux, l'analyse des interactions de classe, le développement plurilittératié et l'acquisition des langues tierces, dans différents contextes de contacts de langues et de cultures. Elle est l'auteure de l'ouvrage Plurilinguismes et école, paru à Paris aux Éditions Didier (Collection LAL, 2006). Elle est actuellement éditeur en chef (pour le français) de la revue en-ligne Child Health and Education/Santé et Éducation de l'Enfance (www.childhealthandeduction.com) et CoRédactrice avec Laura Collins de la Revue Canadienne des Langues Vivantes/Canadian Review of Modern Languages. Elle a co-présidé le Comité scientifique de la revue Recherches et applications - le Français dans le Monde (http://fipf.org/publications/recherches-applications) avec Francis Carton et Geneviève Zarate jusqu'en juillet 2012.

dmoore[at]sfu.ca

http://www.educ.sfu.ca/profiles/?page_id=211 
Simon Fraser University, Faculty of Education, 8888 University Drive, Burnaby BC V5A 1S6, Canada. 